\title{
Sensor nodes selection in Wireless Sensor Networks over a rich scattering environment
}

\author{
Maha Ben Zid \\ UJF-Grenoble I / ENIT \\ GIPSA-Lab - UMR 5216 CNRS \\ France / Tunisia \\ maha.ben-zid@gipsa-lab.grenoble-inp.fr
}

\author{
Kosai Raoof \\ UJF-Grenoble I \\ GIPSA-Lab - UMR 5216 CNRS \\ France \\ kosai.raoof@gipsa-lab.grenoble-inp.fr
}

\author{
Ammar Bouallègue \\ ENIT \\ 6'Com-Lab \\ Tunisia \\ ammar.bouallegue@enit.rnu.tn
}

\begin{abstract}
This paper is concerned with the sensor nodes selection in Wireless Sensor Networks (WSNs). This technique is inspired from antennas selection in the Multiple Input Multiple Output (MIMO) systems. Antennas selection scheme stills be required in Wireless Sensor Networks. In fact, the deployed sensor nodes in the network are limited in power and thus antennas selection processing is needed for energy saving and lifetime maximizing. In this paper, we introduce the sensor nodes selection processing and we present an algorithm for nodes selection in WSNs. The suggested algorithm is based on Signalto-Noise Ratio (SNR) maximizing and is tested over the realistic rich scattering transmission environment.
\end{abstract}

\section{INTRODUCTION}

Recent advances in Distributed Sensor Networks (DSNs) [1] have enabled the emergence of very flexible wireless network design with high scalability. Typical candidate for these networks is the famous Wireless Sensor Network (WSN). WSN [2], [3] consists of heterogeneous and single autonomous sensor nodes which are densely deployed in the area of interest. This dense deployment can be leveraged by the envisioned applications which are intended to monitor a variety of ambient conditions such as pressure, temperature and sound. Design of WSNs is challenging since sensing data require to be acheminated over a long distance using individual power-constrained sensor nodes [4] associated with very limited lifetime batteries. Wide dense populated sensor networks can be deployed in the form of groups of nodes called clusters [5], [6], [7]. The groups of sensor nodes generally consist of a set of sensor nodes with a coordinator node called cluster head. Multi-hop transmission has been adopted by the WSNs by structuring the network into pairs of clusters called hops. Each pair of clusters (See Fig.1) could be assimilated to a virtual distributed Multiple Input Multiple Output (MIMO) system [8], [9], [10], [11], [12], [13].

\section{Motivation}

Several recent papers have focused on processing techniques for MIMO systems in the context of WSNs. This paper is devoted to antennas selection processing for WSNs. We assume that each sensor node is equipped with a single antenna. As such, antennas selection in WSNs is confused with sensor nodes selection. One motivation for antennas

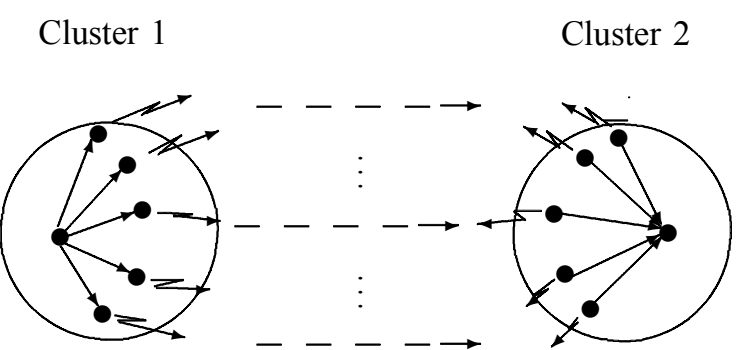

Fig. 1. A pair of cluster nodes

selection within WSNs is that sensor nodes operate on small batteries which are difficult to replace [14]. Optimal resources allocation is then needed since sensors have limited available energy and need to perform a wide range of demanding tasks [15]. Hence, it is worthwhile to optimize the use of the sensor nodes by selecting the appropriate subsets of sensor nodes over the groups of nodes in the network. The antennas selection technique performed for MIMO systems will be firstly discussed in this paper.

\section{Antennas SELECTION: AN OVERVIEW}

Antennas selection techniques [16], [17] have been firstly proposed for the conventional MIMO systems (Fig.2).

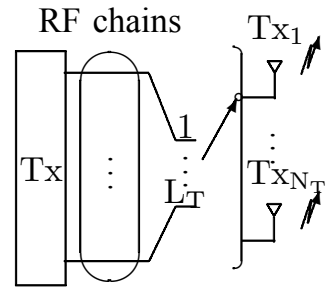

Transmitter

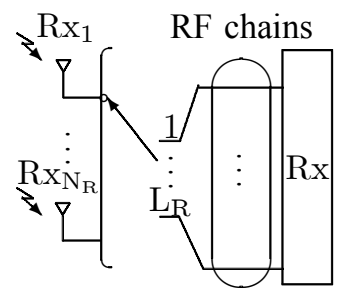

Receiver
Fig. 2. Antenna selection in conventional MIMO system

Antennas selection has been applied in system design in order to reduce the system complexity and cost. In fact, the deployment of multiple antennas would require the implementation of multiple Radio Frequency (RF) chains which could be costly in terms of size, power and hardware [18]. The idea of antennas selection is to select $\mathrm{L}_{\mathrm{T}}$ antennas among the 
$\mathrm{N}_{\mathrm{T}}$ transmit antennas $\mathrm{Tx}_{1}, \ldots, \mathrm{Tx}_{\mathrm{N}_{\mathrm{T}}}$ and / or $\mathrm{L}_{\mathrm{R}}$ antennas among the $\mathrm{N}_{\mathrm{R}}$ receive antennas $\mathrm{Rx}_{1}, \ldots, \mathrm{Rx}_{\mathrm{N}_{\mathrm{R}}}$ as depicted in Fig.2. Antennas selection evidently reduces the channel capacity for MIMO systems. Nevertheless, antennas selection should be optimal in the manner that subsets of antennas are that ones that allow for the best performances in terms of system capacity and reliability of the transmission link. When $\mathrm{L}_{\mathrm{T}}$ antennas are selected at the transmitter and $\mathrm{L}_{\mathrm{R}}$ antennas are selected at the receiver, the associated channel will be denoted $\mathbf{H}_{\mathrm{S}}\left(\mathrm{L}_{\mathrm{R}} \times \mathrm{L}_{\mathrm{T}}\right)$. The antennas selection in MIMO systems is then intended to maximize the channel capacity which is expressed as :

$$
\begin{aligned}
\mathrm{C}\left(\mathbf{H}_{\mathrm{S}}\right) & =\log _{2}\left[\operatorname{det}\left(\mathrm{I}_{\mathrm{L}_{\mathrm{T}}}+\frac{\mathrm{SNR}}{\mathrm{L}_{\mathrm{R}}} \mathbf{H}_{\mathrm{S}}^{\mathrm{H}} \mathbf{H}_{\mathrm{S}}\right)\right] \\
& =\log _{2}\left[\operatorname{det}\left(\mathrm{I}_{\mathrm{L}_{\mathrm{R}}}+\frac{\mathrm{SNR}}{\mathrm{L}_{\mathrm{T}}} \mathbf{H}_{\mathrm{S}} \mathbf{H}_{\mathrm{S}}^{\mathrm{H}}\right)\right]
\end{aligned}
$$

$(\cdot)^{\mathrm{H}}$ denotes the conjugate transpose operator.

Ergodic capacity of MIMO system with antennas selection at the transmitter and the receiver is shown in Fig. 3. The notation ASel refers to Antennas Selection. MIMO transmission is carried over a Rayleigh MIMO channel with Additive White Gaussian Noise (AWGN).

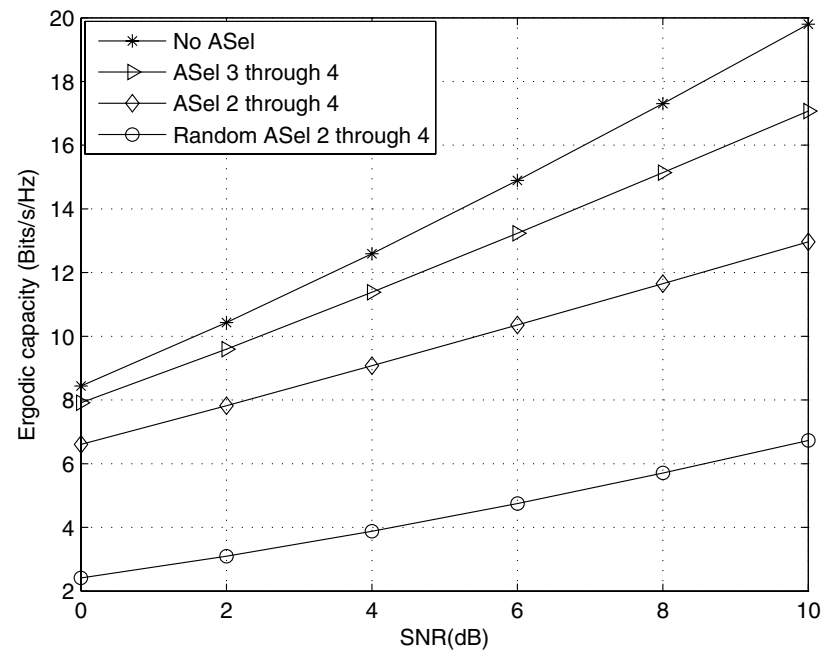

Fig. 3. Impact of antennas selection on the ergodic MIMO system capacity

Plotted curves depict the ergodic capacity for different numbers of selected antennas. The adopted MIMO system is equipped with 4 antennas at both ends of the transmission link. We assume that the same number of antennas is selected at both the transmit and the receive ends. Four cases are tested by simulation. The ergodic capacity for the MIMO system is visibly affected by antennas selection. However, we can achieve optimal system capacity when performing the antennas selection algorithm. Comparing the curve for the ergodic capacity when two antennas are selected among
4 antennas in the case when antennas selection algorithm is exploited to that one when only random antennas selection is performed, we remark that important improvement in channel capacity is obtained. In addition, optimal antennas selection is shown to achieve a gain of almost $10 \mathrm{~dB}$ in SNR as depicted in Fig. 3.

\section{RICH SCATTERING ENVIRONMENT}

We consider in this section, the concept of scattering in the propagation environment. We call a geometrically based channel model in presence of finite number of scatterers. This model is realistic and would be necessary to perform in our case. In fact, sensor nodes at the transmit side are far away from that ones at the receive side and the effect of scatterers in the propagation environment could not be neglected. Rich scattering environment is sketched in Fig. 4.

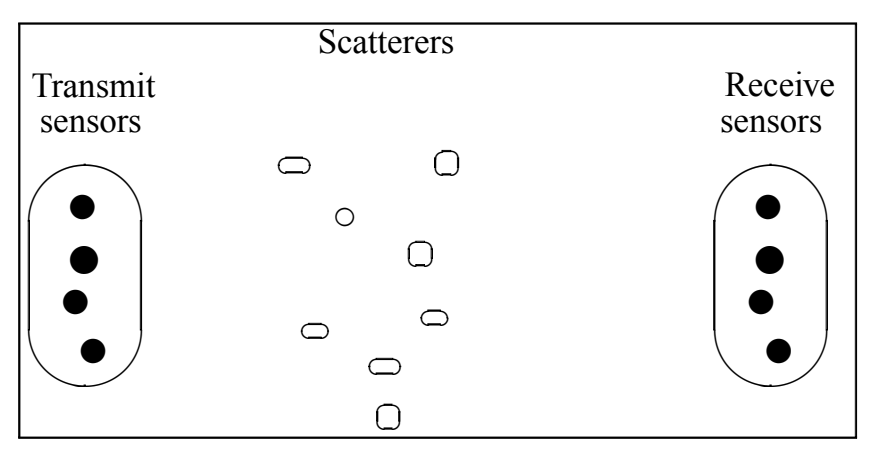

Fig. 4. Rich scattering environment

Scattering phenomena leads to angle spreading at both the azimuthal and the elevation directions. Angle spreading refers to the spread of Direction of Arrival (DOA)/Direction of Departure (DOD) of the multipath components at the transmit/receive antennas. In Fig. 5, the angle spreads at both the transmit side and the receive side are respectively denoted by $\Theta_{T}$ and $\Theta_{R}$. The sensor nodes at the ends of the communication link are denoted by $\mathrm{S}_{\mathrm{Tx}}$ and $\mathrm{S}_{\mathrm{Rx}}$. We assume in this paper, that both the azimuthal and the elevation components of AOAs/AODs of the multipath channels are uniformly distributed.

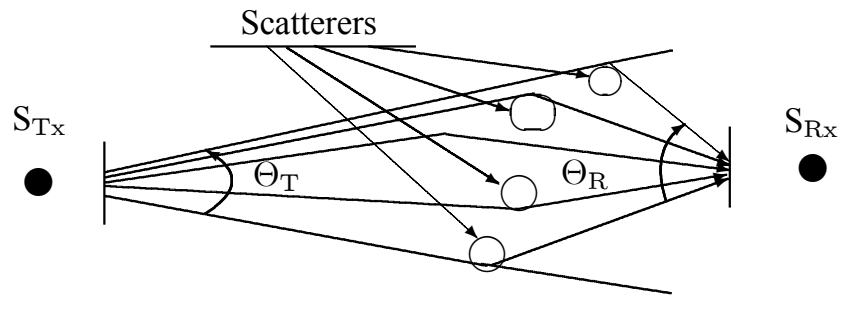

Fig. 5. Angle spreading in rich scattering environment

\section{FINITE SCATTERERS CHANNEL MODELING}

We argue that the finite scatterers model [19] is suitable for modeling the channel relating the sensor nodes at both ends of the communication link. We assume that $\mathrm{N}_{\mathrm{S}}$ scatterers are 
randomly distributed far away from the sensor nodes located at both the transmit and the receive sides of the communication link. The signals travel from the transmit sensor nodes to the receive sensor nodes via a number of multipath components. Each path specifies a Direction of Departure (DOD) from the transmitting sensor node and a Direction of Arrival (DOA) at the receiving sensor node. In 3-Dimensional (3D) geometric model, each DOD, $\Phi_{\mathrm{T}}=\left(\theta_{\mathrm{T}}, \phi_{\mathrm{T}}\right)$ and DOA, $\Phi_{\mathrm{R}}=\left(\theta_{\mathrm{R}}, \phi_{\mathrm{R}}\right)$ is defined by both the elevation and azimuthal components $\left(\theta_{\mathrm{T}}, \phi_{\mathrm{T}}\right)$ at the transmit side and $\left(\theta_{\mathrm{R}}, \phi_{\mathrm{R}}\right)$ at the receive side. We distinguish the elevation angle spread (ES) and the azimuth angle spread (AS). The ES and the AS respectively refer to the maximum deviation of the angle spread from the Mean Angle of Arrival (MAOA) and the Mean Angle of Departure (MAOD)[20]. We assume that the azimuth/elevation angles of the multipath channels are uniformly distributed over the range $\left[\mathrm{MAS}-\mathrm{A}_{\mathrm{S}} \mathrm{MAS}+\mathrm{A}_{\mathrm{S}}\right.$ ] $\left(\mathrm{A}_{\mathrm{S}}\right.$ : Angle Spread, MAS: Mean Angle Spread). In this paper, we concentrate our analysis on the uniform distribution of the angle spread.

Sensor nodes are randomly deployed at both the transmitting and the receiving sides. We assume any geometric location for sensor nodes. The discrete channel model with $\mathrm{N}_{\mathrm{S}}$ scatterers is then:

$$
\begin{aligned}
\mathbf{H} & =\frac{1}{\sqrt{N_{\mathrm{S}}}} \sum_{\mathrm{n}=1}^{\mathrm{N}_{\mathrm{S}}} \mathbf{a}_{\mathrm{R}}\left(\Phi_{\mathrm{R}, \mathrm{n}}\right) \cdot \beta_{\mathrm{n}} \cdot \mathbf{a}_{\mathrm{T}}^{\mathrm{H}}\left(\Phi_{\mathrm{T}, \mathrm{n}}\right) \\
& =\frac{1}{\sqrt{\mathrm{N}_{\mathrm{S}}}} \mathbf{A}_{\mathrm{R}} \cdot \mathbf{B} \cdot \mathbf{A}_{\mathrm{T}}^{\mathrm{H}}
\end{aligned}
$$

- $\Phi_{\mathrm{T}, \mathrm{n}}$ and $\Phi_{\mathrm{R}, \mathrm{n}}$ respectively define the transmit direction and the receive direction relative to the path component of index $\mathrm{n}$.

- $\beta_{\mathrm{n}}, \mathrm{n}=1, \ldots, \mathrm{N}_{\mathrm{S}}$ is a random complex amplitude of the n-th path

The matrices $\mathbf{A}_{\mathrm{R}}\left(\mathrm{K} \times \mathrm{N}_{\mathrm{S}}\right), \mathbf{B}\left(\mathrm{N}_{\mathrm{S}} \times \mathrm{N}_{\mathrm{S}}\right)$ and $\mathbf{A}_{\mathrm{T}}\left(\mathrm{P} \times \mathrm{N}_{\mathrm{S}}\right)$ are defined as:

- $\mathbf{A}_{\mathrm{R}}=\left[\mathbf{a}_{\mathrm{R}}\left(\Phi_{\mathrm{R}, 1}\right), \cdots, \mathbf{a}_{\mathrm{R}}\left(\Phi_{\mathrm{R}, \mathrm{N}_{\mathrm{S}}}\right)\right]$

- $\mathbf{B}=\operatorname{diag}\left(\beta_{1}, \cdots, \beta_{\mathrm{N}_{\mathrm{S}}}\right)$

- $\mathbf{A}_{\mathrm{T}}=\left[\mathbf{a}_{\mathrm{T}}\left(\Phi_{\mathrm{T}, 1}\right), \cdots, \mathbf{a}_{\mathrm{T}}\left(\Phi_{\mathrm{T}, \mathrm{N}_{\mathrm{S}}}\right)\right]$

$\mathbf{A}_{\mathrm{R}}$ and $\mathbf{A}_{\mathrm{T}}$ are respectively composed of the receive steering vectors $\mathbf{a}_{\mathrm{R}}\left(\Phi_{\mathrm{R}, 1}\right), \cdots, \mathbf{a}_{\mathrm{R}}\left(\Phi_{\mathrm{R}, \mathrm{N}_{\mathrm{S}}}\right)$ and the transmit steering vectors $\mathbf{a}_{\mathrm{T}}\left(\Phi_{\mathrm{T}, 1}\right), \cdots, \mathbf{a}_{\mathrm{T}}\left(\Phi_{\mathrm{T}, \mathrm{N}_{\mathrm{S}}}\right)$.

We assume that $\mathrm{P}$ sensor nodes and $\mathrm{K}$ sensor nodes are respectively distributed at the transmitting side and the receiving side. According to these considerations, for each path component of index $n, n=1, \ldots, N_{S}$, transmitting steering vector [21] $\mathbf{a}_{\mathrm{T}}\left(\Phi_{\mathrm{T}, \mathrm{n}}\right)$ of size $(\mathrm{P} \times 1)$ is expressed as :

$$
\mathbf{a}_{\mathrm{T}}\left(\Phi_{\mathrm{T}, \mathrm{n}}\right)=\left\{\exp \left(-\mathrm{j} \cdot \overrightarrow{\mathrm{K}}_{\mathrm{T}}(\mathrm{n}){\overrightarrow{\mathrm{r}_{\mathrm{T}}}}^{(\mathrm{p})}\right) ; \mathrm{p}=1, \ldots, \mathrm{P}\right\}^{\mathrm{T}}
$$

$(\cdot)^{\mathrm{T}}$ denotes the transpose operator.

- $\overrightarrow{\mathrm{K}_{\mathrm{T}}}(\mathrm{n})$ is the transmit wave vector of amplitude $2 \pi / \lambda(\lambda$ is the wavelength).

- $\overrightarrow{\mathrm{r}}_{\mathrm{T}}(\mathrm{p})$ is the position vector of the transmit sensor node $S_{\mathrm{p}}$. Similarly, the 3D position vector of the transmit sensor node $S_{\mathrm{p}}$ is defined by the elevation and the azimuth angles $\left(\theta_{\mathrm{p}}, \phi_{\mathrm{p}}\right)$ as:

$$
\overrightarrow{\mathrm{r}}_{\mathrm{T}}^{(\mathrm{p})}=\mathrm{r}_{\mathrm{T}}^{(\mathrm{p})}\left[\begin{array}{lll}
\sin \left(\theta_{\mathrm{p}}\right) \cos \left(\phi_{\mathrm{p}}\right) & \sin \left(\phi_{\mathrm{p}}\right) \cos \left(\phi_{\mathrm{p}}\right) & \cos \left(\theta_{\mathrm{p}}\right)
\end{array}\right]^{\mathrm{T}}
$$

$\mathrm{r}_{\mathrm{T}}^{(\mathrm{p})}$ is the radial distance to the origin.

At the receive side of sensor nodes, $\mathbf{a}_{\mathrm{R}}\left(\Phi_{\mathrm{R}, \mathrm{n}}\right)$ of size $(\mathrm{K} \times 1)$ is similarly derived as :

$\mathbf{a}_{\mathrm{R}}\left(\Phi_{\mathrm{R}, \mathrm{n}}\right)=\left\{\exp \left(-\mathrm{j} \cdot \overrightarrow{\mathrm{K}_{\mathrm{R}}}(\mathrm{n}){\overrightarrow{\mathrm{r}_{\mathrm{R}}}}^{(\mathrm{k})}\right) \quad ; \mathrm{k}=1, \ldots, \mathrm{K}\right\}^{\mathrm{T}}$

- $\overrightarrow{\mathrm{K}_{\mathrm{R}}}(\mathrm{n})$ is the the receive wave vector.

- $\overrightarrow{\mathrm{r}}^{(\mathrm{k})}$ is the vector position of the receive sensor node $S_{\mathrm{k}}$.

\section{SENSOR NODES SELECTION}

Our goal is to perform sensor nodes selection among the sensor nodes at the receive side and the transmit side. We start by performing the sensor nodes selection algorithm by maximizing the overall channel capacity for the proposed scenario with distributed sensor nodes at the transmitting and the receiving sides of the transmission link. The proposed communication system performs the communication model similar to that one performed by conventional MIMO systems. The mathematical model of the communication system when neglecting the noise signal is then :

$$
\mathbf{y}=\mathbf{H} \cdot \mathbf{x}
$$

- $\mathbf{H}$ is the $(\mathrm{K} \times \mathrm{P})$ complex channel matrix which links the $\mathrm{P}$ transmit sensor nodes to the $\mathrm{K}$ receive sensor nodes.

- $\mathbf{x}=\left[\mathrm{x}_{1}, \ldots, \mathrm{x}_{\mathrm{P}}\right]^{\mathrm{T}}$ is the vector for the transmitted signals.

- $\mathbf{y}=\left[\mathrm{y}_{1}, \ldots, \mathrm{y}_{\mathrm{K}}\right]^{\mathrm{T}}$ is the vector for the received signals at the receive sensor nodes.

The overall channel capacity is derived according to equation (1).

\section{SimUlation RESUlTS FOR SENSOR NODES SELECTION BASED ON CAPACITY MAXIMIZATION}

We consider, in the following 6 sensor nodes at both ends of the transmission link. We assume that sensor nodes are deployed in the $(\mathrm{x}, \mathrm{y})$ plane in a sensing area of $(100 \mathrm{~m})^{2}$. A simulation for randomly generating the geometric positions of the transmit sensor nodes is shown in Fig. 6. Sensor nodes at the receive side are generated in a similar way but are assumed to be located far away of the transmit sensor nodes.

The Angle Spread is set to $90^{\circ}$ and is assumed to be the same for both the AOAs and the AODs. We propose to select 3 sensor nodes among the transmit sensor nodes and also 3 sensor nodes among the receive sensor nodes. The possible subsets of sensor nodes are generated from a binomial distribution. Monte Carlo simulation for the overall system capacity over a rich scattering environment with 20 multipath components is depicted in Fig. 7. The overall system capacity is improved thanks to the optimal sensor nodes selection. 


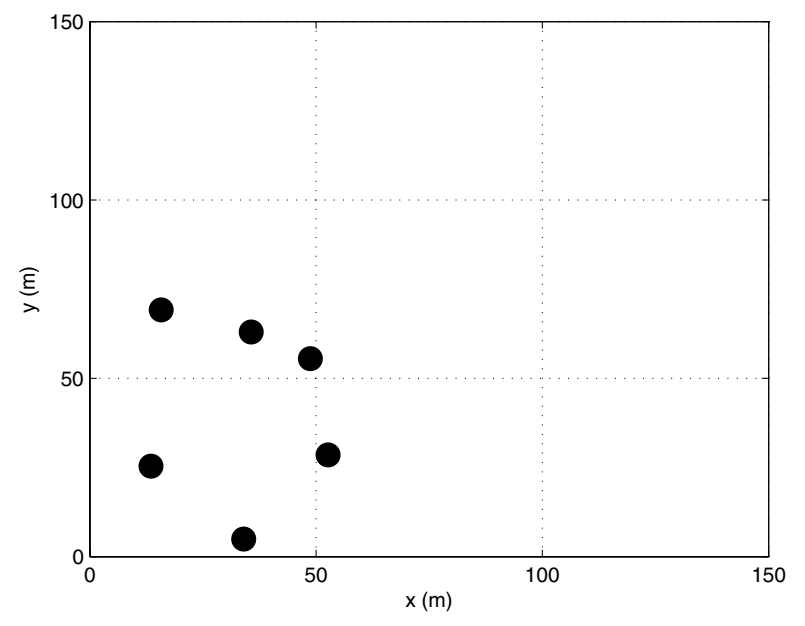

Fig. 6. Transmit sensor nodes

Optimal algorithm for sensor nodes selection reduces the losses in the overall system capacity if sensor node selection is targeted to the appropriate sensor nodes in the network. In addition, we measure important gain in system capacity when comparing optimal sensor nodes selection strategy to random sensor nodes selection. This gain reaches a level up to 1.7 and is shown to be insensible to the SNR.

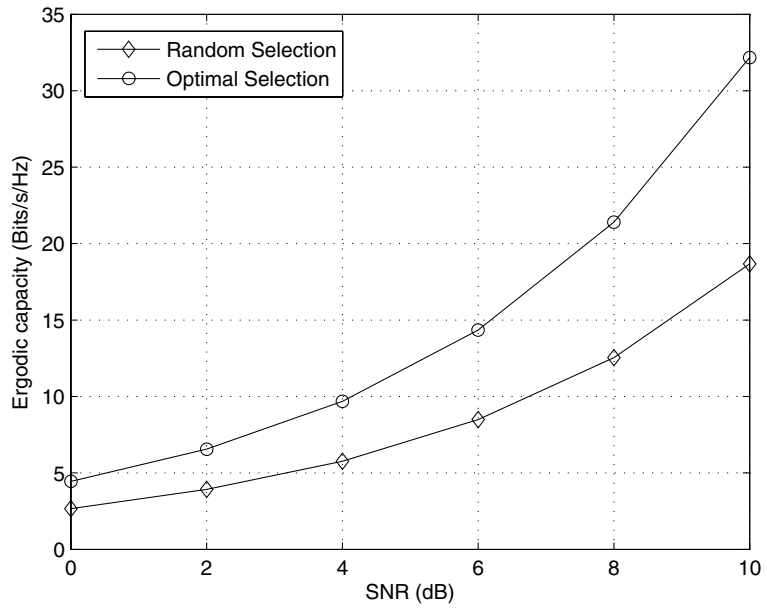

Fig. 7. Sensor nodes selection based on capacity maximization

\section{SENSOR NODES SELECTION : SNR MAXIMIZATION AT THE RECEIVING CLUSTER HEAD}

We examine the impact of the proposed algorithm for the sensor nodes selection on the output SNR at the cluster head sensor at the receive side. We assume independent and identically distributed (i.i.d) channel link between the K sensor nodes at the receive side and the cluster head sensor node. The cluster head sensor node combines the receive sensing incoming signals from the $\mathrm{K}$ sensor nodes.
We assume equal additive noise powers. The output SNR at the cluster head sensor node will be denoted $\Upsilon$ and is expressed as :

$$
\Upsilon=\gamma \cdot\left\|\mathbf{H}_{\mathrm{S}}\right\|_{\mathrm{F}}^{2}
$$

- $\left\|\mathbf{H}_{\mathrm{S}}\right\|_{\mathrm{F}}$ is the Frobenius norm of the associated channel matrix $\mathbf{H}_{\mathrm{S}}$.

- $\gamma$ is the Signal-to-Noise Ratio per sensor node.

\section{Simulation Results}

We perform Monte carlo simulation in order to study the impact of the performed nodes selection algorithm on the overall SNR. Fig. 8 shows that optimal Signal-to-Noise Ratio, $\Upsilon$ is obtained when performing the sensor nodes selection processing.

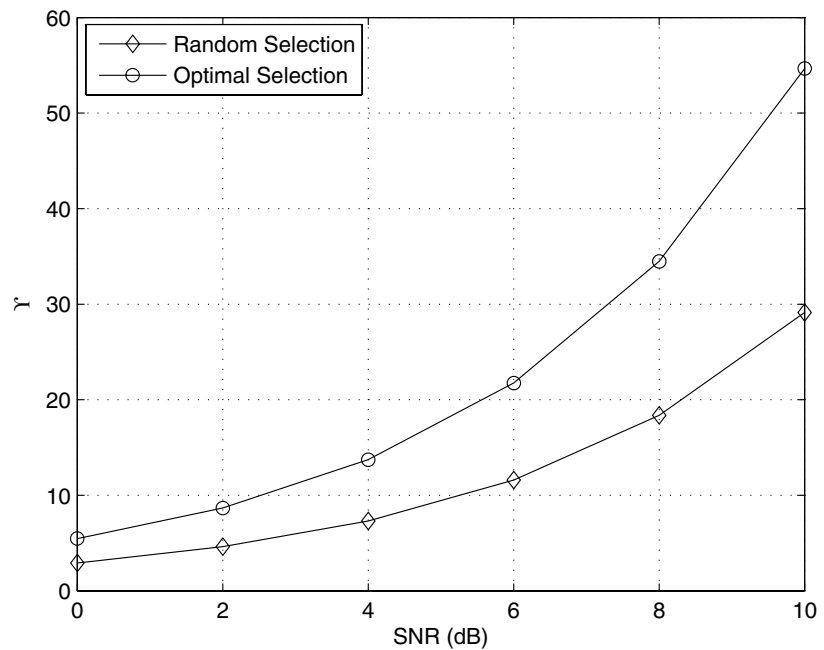

Fig. 8. SNR improvement via optimal sensor nodes selection

\section{Conclusion}

We have analyzed in this paper the sensor nodes selection in WSNs. Our analysis has been addressed for a typical communication system in WSNs when clusters of sensor nodes at both the transmit and the receive sides of the transmission link are randomly distributed at both sensing areas. The geometric channel modeling for rich scattering environment has been studied in this paper. Antennas selection processing has been firstly called for conventional MIMO systems. The selection criteria based on channel capacity maximization has been simulated for the proposed scenario for WSN. The performances of the adopted algorithm were studied in the case of rich scattering environment. The proposed algorithm for sensor nodes selection has been proved to bring improvement in the overall system capacity and to improve the SNR. These results could be very interesting in the context of WSNs since sensor nodes work by limited lifetime battery. Performing adaptive sensor nodes selection among the sensor nodes could 
be beneficial and we can reduce at the best the losses in the overall Signal-to-Noise Ratio thanks to optimal sensor nodes selection strategy.

\section{REFERENCES}

[1] C. -Y. Chong and S. P.Kumar,"Sensor Networks: Evolution, Opportunities, and Challenges", in Proc. IEEE, vol. 91, no. 8, pp. 1247 - 1256, Aug. 2003.

[2] I. Stojmenovic,"Handbook of Sensor Networks: Algorithms and Architectures", John Wiley \& Sons, Inc., ISBN: 9780471684725, Nov. 2005.

[3] Ian F. Akyildiz, W. Su, Y. Sankarasubramaniam and E. Cayirci,"Wireless Sensor Networks: A Survey", Computer Networks Elsevier Journal, vol. 38, no. 4, pp. 393 - 422, Mar. 2002

[4] N. Noordini Nik Abd. Malik, M. Esa, S. Kamilah Syed Yusof and J. Marimuthu,"Optimising of Node Coordination in Wireless Sensor Network", in Proc. PIERS, pp. 1235 - 1238, Moscow, Aug. 2009.

[5] M. R. Anderberg, "Cluster Analysis for Applications", Academic Press, London, Jan. 1973.

[6] S. Bandyopadhyay and E. J. Coyle,"An Energy Efficient Hierarchical Clustering Algorithm for Wireless Sensor Network", in Proc. IEEE INFOCOM, pp. 1713 - 1723, San Francisco, Apr. 2003.

[7] A. del Coso, S. Savazzi, U. Spagnolini and C. Ibars, "Virtual MIMO Channels in Cooperative Multi-hop Wireless Sensor Networks", 40th Annual Conference on Information Sciences and Systems, pp. 75 - 80, Mar. 2006.

[8] M. Ben Zid, K. Raoof and A. Bouallègue, Manuscript: "MIMO Systems and Cooperative Networks Performances", accepted for publishing in : Cognitive radio, Scientific Research Publishing, 2011.

[9] K. Raoof, M. Ben Zid, N. Prayongpun and A. Bouallègue, Manuscript: "Advanced MIMO Techniques: Polarization Diversity and Antenna Selection", accepted for publishing in : MIMO Systems, Theory and Applications, ISBN 978-953-7619-X- X, INTECH, 2011.

[10] O. Ben Rhouma, M. Ben Zid, S. Houcke and A. Bouallègue,"An Iterative Blind Receiver : Implementation and Performance", International Journal of Computer Science and Network Security (IJCSNS), vol.7, no.9, pp. 73 - 78, Sept. 2007.

[11] N. Prayongpun and K. Raoof,"MIMO Channel Capacities in Presence of Polarization Diversity with and without Line-of-Sight Path", Journal WSEAS Transactions on Communications, vol. 5, no. 9, pp. 1744 - 1750 Sept. 2006

[12] K. Raoof and H. Zhou, “Advanced MIMO Systems", Scientific Research Publishing, ISBN : 978-1-935068-02-0, Sept. 2009.

[13] K. Raoof, M.A. Khalighi and N. Prayongpun,"MIMO Systems: Principles, Advanced Polarization and Iterative Techniques", in Adaptive Signal Processing for Wireless Communications, CRC Press, Aug. 2008.

[14] G. N. Bravos and A. G. Kanatas,"Energy efficiency of MIMO-based Sensor Networks with a Cooperative Node Selection Algorithm", IEEE International Conference on Communications, ICC '07, pp. 3218 3223, Glasgow, Aug. 2007.

[15] F. Kerasiotis, A. Prayati, C. Antonopoulos, C. Koulamas and G. Papadopoulos, "Battery Lifetime Prediction Model for a WSN Platform", 4th International Conference on Sensor Technologies and Applications (SENSORCOMM), Venice, Jul. 2010.

[16] A. Ghrayeb,"A Survey on Antenna Selection for MIMO Communication Systems", Information and Communication Technologies, ICTTA '06, pp. 812 - 818, Oct. 2006.

[17] S. Sanayei and A. Nosratinia,"Antenna Selection in MIMO Systems", IEEE Communications Magazine, vol. 42, no. 10, pp. 68 - 73, USA, Oct. 2004.

[18] Q. Trung Dong, N. Prayonpun and K. Raoof,"Antenna Selection for MIMO Systems in Correlated Channels with Diversity Technique", The 4th International Conference on Wireless Communications, Networking and Mobile Computing, China, Nov. 2008.

[19] A. Burr,"Capacity bounds and estimates for the finite scatterers MIMO wireless channel", IEEE Journal On Selected Areas in Communications, vol. 21, no. 5, pp. 812 818, Jun. 2003.

[20] S. K. Yong and J. S. Thompson,"A Three-Dimensional Spatial Fading Correlation Model for Uniform Rectangular Arrays", IEEE Transactions on Wireless Communications, vol. 4, no. 6, pp. 2856 - 2869, Nov. 2005.
[21] M. Steinbauer, A.F Molisch and E. Bonek,"The double-directional radio channel", IEEE Antennas and Propagation Magazine, vol. 43, no. 4, pp. 51 - 63, Aug. 2001. 